Espacio y Desarrollo No 32, 2018, pp. 125-136 (ISSN 1016-9148)

https://doi.org/10.18800/espacioydesarrollo.201802.006

\title{
Sustentos PARA QUE los ACEITES COMESTIBLES RESIDUALES (ACR) SEAN CONSIDERADOS DENTRO DEL RÉGIMEN ESPECIAL DE GESTIÓN DE RESIDUOS DE BIENES PRIORIZADOS DEL PERÚ
}

\author{
Sayda Estela Mujica Bueno \\ smujica@pucp.edu.pe
}

Fecha de recepción: 6 de septiembre de 2018

Fecha de aceptación: 9 de octubre de 2018

\section{Resumen}

En este artículo se presentan los impactos que los aceites comestibles residuales (ACR) están ocasionando tanto en el ambiente como en la salud humana, considerando las exigencias de la Ley 1278 y su reglamento Decreto Supremo 014-2017-MINAM sobre residuos sólidos. Se explica por qué esta ley — cuyo título y orientación se enfoca en los residuos sólidos- es pertinente y aplicable para los aceites que son líquidos, así como qué son bienes priorizados, su régimen y cómo los fines de la ley están relacionados con los ACR. Se repasa la situación de los ACR para cada uno de los principios de la ley. Se presenta el ciclo de vida tradicional de los ACR, así como el ciclo de vida bajo el enfoque de la economía circular. Se da alcances sobre cómo dar valor a los ACR en la producción de biodiésel, bajo el principio de valorización del residuo. Se identifica a los actores responsables y copartícipes bajo los principios de responsabilidad extendida del productor (REP) y de Responsabilidad Compartida. Bajo el Principio de Protección del Ambiente y la Salud Pública, se presentan efectos eco-tóxicos y tóxicos que los ACR generan cuando no son tratados adecuadamente. En el desarrollo más amplio de la REP, se hace hincapié en los actores responsables y se demuestra que los ACR cumplen con los criterios para ser considerados dentro del régimen especial de gestión de residuos de bienes priorizados del Perú.

Palabras clave: aceites comestibles residuales (ACR), biodiésel, gestión de residuos, bienes priorizados, Responsabilidad Extendida del productor (REP).

Reasons to consider waste Cooking Oils (WCO) within the Special Regimen of Waste Management of Prioritized Goods in Peru

\section{Abstract}

In this article, the health and environmental impacts caused by Waste Cooking Oils (WCO) are presented, considering the requirements of Law 1278 and its regulations contained in the Supreme Decree 014-2017-MINAM-PERU for solid waste. This paper explains why this 
regulation - whose title and orientation focuses on solid waste - is relevant and applicable to liquid oils, as well as the concept of prioritized goods, their regimen and how the law's principles are related to WCO. The situation of WCO is reviewed for each of the policies contained in the regulations. The traditional life cycle for WCO is presented, as well as the new life cycle approach under circular economy. The possibility of utilizing WCO in the production of biodiésel is presented in order to give value to the first, under the Principle of valorization of waste. The responsible actors and partners are identified under the principles of Extended Producer Responsibility (REP) and Shared Responsibility. The eco-toxic and toxic effects that WCO represent, when inadequately managed, are identified, under the principle of Protection of the Environment and Public Health. In the broader development of the REP, this work emphasizes the role of responsible actors and demonstrates that WCO meets the criteria to be considered within the special regime for prioritized goods.

Keywords: Waste cooking oil (WCO), biodiesel, waste management, prioritized goods, Extended Producer Responsibility (REP).

\section{INTRODUCCIÓN}

A la luz de la nueva reglamentación para los residuos sólidos por medio del Decreto legislativo (DL) 1278 que aprueba la nueva Ley de Gestión Integral de Residuos Sólidos y su reglamento Decreto supremo 014-2017-MINAM - que también rige para los aceites comestibles residuales_ - es posible identificar los argumentos bajo los cuales se justifica por qué determinados productos pueden ser considerados dentro del Régimen Especial de Gestión de Residuos de Bienes Priorizado de dicha ley.

En este trabajo se presenta la situación de los ACR frente a los contenidos de la ley y del reglamento, así como algunas de las prácticas locales relacionadas. Primero se explica la razón por la que, a los aceites, que son líquidos, se les está considerando en una ley para sólidos; luego se define el concepto de bienes priorizados, se presentan los principios contemplados en la ley relacionándolos con los ACR; así como los alcances de responsabilidad extendida del productor para el caso de los ACR, para al final presentar los criterios del reglamento bajo los cuales se determina en qué casos los residuos pueden ser considerados bienes priorizados y cómo estos criterios son abordados y justifican que los ACR sean considerados dentro del «Régimen Especial de Gestión de Residuos de Bienes Priorizados».

\section{Sustentos}

\subsection{Competencia de la ley y su reglamento para los ACR que son líquidos}

En el único anexo de la ley que se refiere a definiciones para residuos sólidos, se indica: «Los residuos sólidos incluyen todo residuo o desecho en fase sólida o semisólida. También se considera residuos aquellos que siendo líquido o gas se encuentran 
contenidos en recipientes o depósitos que van a ser desechados, así como los líquidos o gases, que por sus características fisicoquímicas no puedan ser ingresados en los sistemas de tratamiento de emisiones y efluentes y por ello no pueden ser vertidos al ambiente. En estos casos los gases o líquidos deben ser acondicionados de forma segura para su adecuada disposición final» (Ley 1278, 2016).

Y en el reglamento del DL 1278 (DS 014, 2017) en la Lista B: Residuos no peligrosos del Anexo V se presentan a las grasas y aceites con el numeral B3065: "Grasas y aceites comestibles de origen animal o vegetal para desecho (p. ej. aceite de freír), siempre que no exhiban las características del Anexo IV lista de características peligrosas». De acuerdo a la Resolución legislativa 26234, Convenio de Basilea, (RL 26234,1993) a menos que contengan materiales o sustancias, que son establecidos en el Anexo I del Convenio de Basilea, en una cantidad tal que les confiera una de las características del Anexo IV, lista de características peligrosas, como vemos este párrafo del reglamento presenta como ejemplo a los aceites de freír, que son objeto de esta presentación.

Por lo tanto, los ACR son líquidos regulados por esta ley y son considerados residuos no peligrosos ubicados en la lista B del reglamento.

\subsection{Qué se entiende por bienes priorizados}

En el anexo I del reglamento (DS 014, 2017) sobre Definiciones se explica: «Son aquellos bienes que requieren un manejo especial al momento de convertirse en residuo, ya que estos pueden ser valorizados o requieren manejo diferenciado para su disposición final. A estos bienes se le aplica el Principio de Responsabilidad Extendida del Productor, haciendo responsable al productor de los bienes en todo el Ciclo de Vida del Producto». La situación de los ACR que a continuación se presenta deja en claro que los ACR caen dentro de esta definición.

\subsection{Respecto a la finalidad de la ley}

La ley enuncia como su finalidad: "primero la prevención o minimización de la generación de residuos sólidos en el origen, frente a cualquier otra alternativa. En segundo lugar... la recuperación y la valorización material y energética de los residuos, siempre que se garantice la protección de la salud y del medio ambiente» (Ley 1278, 2016).

Sobre lo primero, los ACR son residuos de las actividades de cocción de alimentos en los restaurantes, los hogares y por los ambulantes que ofrecen alimentos fritos. Estos actores son los que deben ser capacitados en el manejo de los ACR para la prevención y minimización de la generación de estos residuos en su origen.

Para lo segundo, la recuperación y la valorización material y energética de los residuos para el caso de los ACR, consiste en un manejo especial (que comprende una planta de acopio) diferente a la de los servicios de baja policía y de parques y jardines de la muni- 
cipalidad; luego de lo cual estos residuos entrarían en diversos procesos productivos, que conllevaría su valorización en una planta de producción. Todo esto supone el diseño de un sistema de manejo especial, como se indica en el reglamento (DS 014, 2017) para el caso de bienes priorizados y se explica en el subtítulo 2.5 párrafo e) de este escrito.

\subsection{Respecto a los principios que menciona ley}

\subsubsection{El principio de economía circular}

Según el modelo de economía tradicional, el ciclo de vida de los aceites comestibles comprende las etapas de cultivo de la materia prima, la producción de los aceites, la comercialización, el uso de los aceites y la disposición de los residuos ACR al ambiente, como se muestra en la figura 1. Sin embargo, siguiendo el interés económico que los ACR generan, acorde con la economía circular, se ha comenzado a incorporar dentro de su ciclo de vida más actividades que permiten al mismo tiempo, dar disposición adecuada de los residuos y generar valor agregado, de modo que el ciclo de vida tradicional (ver figura 1) cambie, añadiendo las etapas de recolección de los residuos, el acopio de los mismos y su tratamiento o transformación para obtener otros productos, como es el caso de la transformación de los ACR en biodiésel que se ilustra en la figura 2. El hecho de añadir estas últimas actividades al ciclo de vida tradicional de los ACR cumpliría con la regeneración y recuperación de los recursos y no se limitaría solo a la obtención de bienes.

\subsubsection{El principio de la valorización de residuos}

Como se muestra en el párrafo anterior, los ACR presentan potencial como recurso económico y por lo tanto no deben ser destinados a los rellenos sanitarios o a la disposición final. Por lo contrario, se debería priorizar su valorización en actividades de reciclaje como la generación de energía por medio de la producción de biodiésel; más aún para cumplir con la reglamentación local, según la cual el diésel que se vende en el Perú debe contener un 5\% de biodiésel el cual se importa.

\subsubsection{El principio de responsabilidad extendida del productor (REP)}

La ley (Ley 1278, 2016) identifica a los fabricantes, importadores, distribuidores y comercializadores como los responsables de participar en las etapas del ciclo de vida de un producto, además de cumplir con la ecoeficiencia, minimizar la generación de residuos y/o facilitar su valorización; y un actuar sostenible. Son las empresas que fabrican, importan y/o comercializan los aceites comestibles vegetales, quienes bajo este principio deben involucrarse en las etapas del ciclo de vida de estos aceites, para que su ciclo de vida sea sostenible. Este punto va a ser analizado en mayor detalle en el punto 2.5 sobre responsabilidad extendida del productor. 
Figura 1. Ciclo de vida tradicional de los ACR

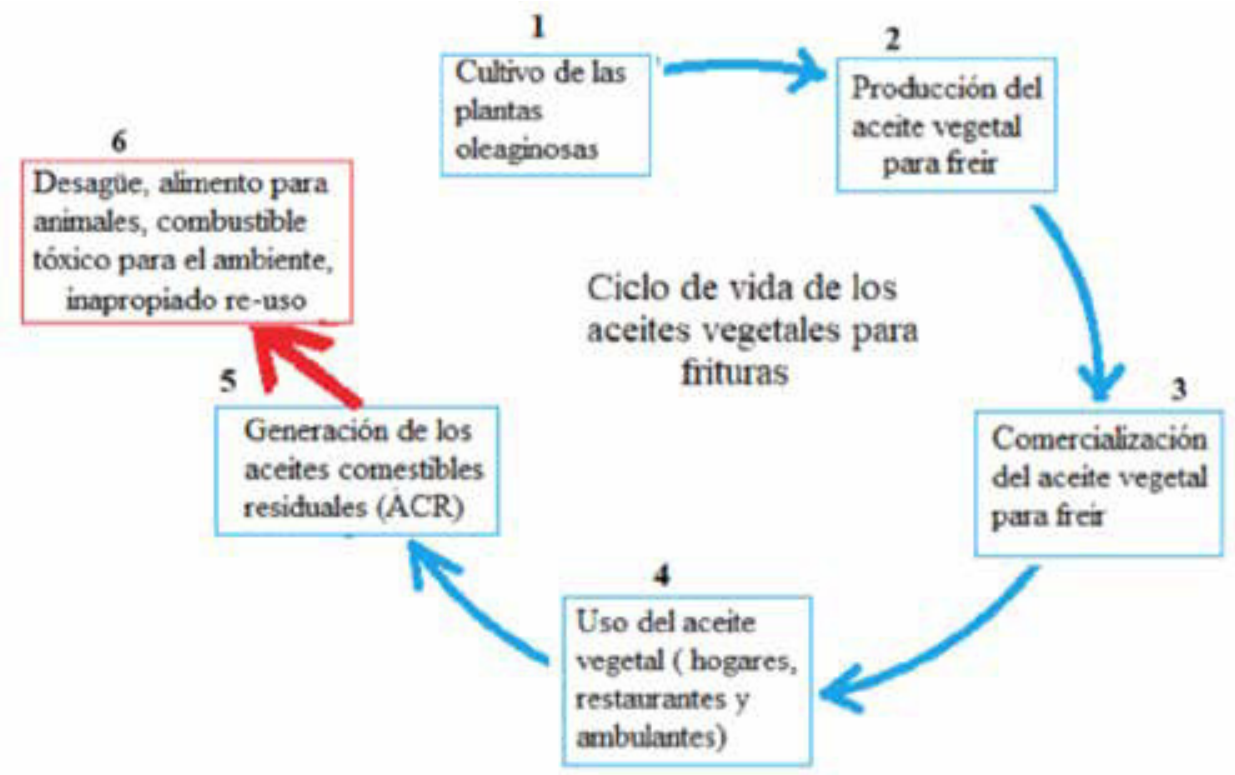

Figura 2. Ciclo de vida de los ACR de acuerdo con la economía circular

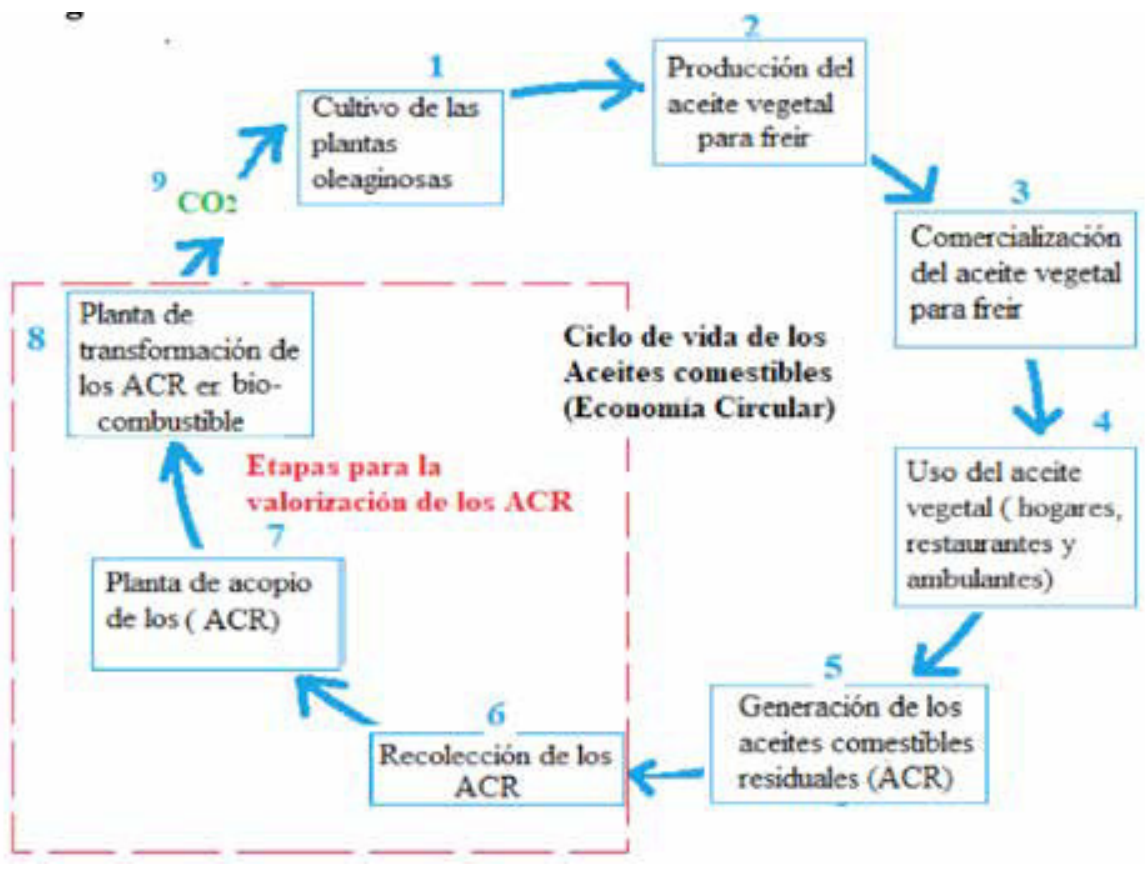




\subsubsection{El principio de responsabilidad compartida}

Bajo este principio se indica que hay «una corresponsabilidad social» en la que de manera «conjunta, coordinada y diferenciada» deben participar «los generadores, operadores de residuos y municipalidades» (Ley 1278, 2016). Para el caso de los ACR, esto conlleva incorporar a los restaurantes, hogares y ambulantes, empresas operadoras de residuos sólidos que estén acopiando y/o procesando los ACR en otros productos, siendo entonces todos ellos, junto con la municipalidad, copartícipes de esta responsabilidad.

\subsubsection{El principio de protección del ambiente y la salud pública}

Los ACR, al ser vertidos al desagüe, pueden llegar a las fuentes de agua subterránea, ríos y lagos y contaminarlas. De llegar a las plantas de tratamiento de aguas encarecen dicho tratamiento: un litro de aceite puede contaminar hasta 40000 litros lo que equivale al consumo de agua de una persona en su domicilio. Estudios en España hacen notar que la depuración de un litro de ACR tiene un costo de 0,45 euros/ litro, que comparado con los costos promedio de tratamiento es 700 veces más caro (Gonzáles y Gonzáles, 2015). Así también pueden llegar a deteriorar la calidad del suelo y su fertilidad.

En ocasiones, el aceite usado es comercializado como combustible debido a su alto poder calorífico; pero la inadecuada combustión ocasiona la contaminación del aire por la emisión de gases tóxicos y consecuentes daños a la salud y al ambiente (Guillén y Uriarte, 2012). Estas características pueden llevar a que los ACR sean considerados ecotóxicos y caigan dentro de la lista de los productos con características peligrosas, ubicándose en la clase 9 de la clasificación de las Naciones Unidas (RL 26234,1993), y que el reglamento de la ley (DS 014, 2017) recoge en el anexo IV con el código H12 - Ecotóxico, definido como «sustancias o residuos que, si se liberan, tienen o pueden tener efectos adversos inmediatos o retardados en el medio ambiente, debido a la bioacumulación o los efectos tóxicos en los sistemas bióticos». Estas mismas características químicas (Guillén y Uriarte, 2012) pueden situar a los ACR dentro de la clase 6.1 con código H6.1 Tóxicos Venenos Agudos de la lista de los productos con características peligrosas de las Naciones Unidas (RL 26234,1993) y que el reglamento de la ley lo presenta en el anexo IV (DS 014, 2017). Los H6.1 están definidos como: «Sustancias o residuos que pueden causar la muerte o lesiones graves o daños a la salud humana, si se ingieren o inhalan o entran en contacto con la piel» (RL 26234,1993). Por lo que las autoridades de salud en el mundo dan normas al respecto. En el Perú, para los ACR el Ministerio de Salud ha ordenado el 2014 por Resolución Ministerial la modificación del artículo 24 de la Norma Sanitaria para el Funcionamiento de Restaurantes y Servicios Afines, la que en el artículo 1 apartado b) sobre el proceso de cocción indica:

las grasas y aceites utilizados para freír no deben calentarse a más de $180^{\circ} \mathrm{C}$ y durante su reutilización deben filtrarse para eliminar partículas de alimentos que hubieran quedado 
de las frituras anteriores. Cuando los cambios de color, olor, turbidez, sabor, entre otros den indicios de un recalentamiento excesivo o quemado, deben desecharse. Con fines de control de calidad de los aceites y grasas reutilizados en la elaboración de frituras, se consideran como no aptos para el consumo humano, debiendo desecharse cuando contienen más del 25\% de compuestos polares (RM 965, 2014).

También en el anexo 3 de la ficha para la evaluación de la norma sanitaria del Ministerio de Salud para el funcionamiento de restaurantes y servicios afines por medio de la Resolución ministerial del año 2005, en el punto sobre Preparación (RM 363-2005/ MINSA) se indica: «el aceite debe tener un aspecto limpio del aceite utilizado, color ligeramente amarillo y sin olor a rancio». Como se puede notar ya se está dando un tratamiento especial y normas para el uso de los aceites que vienen del sector salud, lo que hace pensar en la interacción entre los sectores de salud y ambiente, bajo un sistema especial de manejo de los ACR, que involucre a los centros de abastecimiento de comidas, los hogares y ambulantes para proteger la salud individual y colectiva de las personas y vivir en un ambiente equilibrado y adecuado para el desarrollo de la vida.

\subsection{Responsabilidad extendida del productor}

La responsabilidad extendida del productor (REP) surge como una estrategia de protección ambiental que tiene por objetivo alcanzar un impacto disminuido por cada producto haciendo responsable al productor por todo su ciclo de vida (Tojo, 2004). El concepto de responsabilidad extendida del productor se genera de la interpretación de dos principios internacionales del derecho ambiental: (i) el principio de prevención y (ii) el principio contaminador- pagador (Dulanto, 2017). Es por esto que dicha ley en el título III, artículo del 12 al 14 (Ley 1278, 2016) anota: «Los fabricantes, importadores, distribuidores y comerciantes se involucran activamente, según corresponda, a lo largo de las diferentes etapas del ciclo de vida del producto, participar de uno o más procesos del manejo de los residuos sólidos, priorizando su recuperación y valorización». Para el caso de los ACR, el ciclo de vida que considera su valorización incluye mayormente a tres actores más: los recolectores, acopiadores y los que transforman los ACR. Estas actividades se muestran en la figura 2. Se entendería que los demás actores involucrados deben extender su responsabilidad a las actividades de recolección, acopio y transformación y/o valorización. Además, por el principio de Responsabilidad Compartida, los restaurantes, hogares, ambulantes, empresas operadoras de residuos sólidos y la municipalidad son copartícipes de la responsabilidad. De este modo, se tiene a los responsables y a los coparticipes como los actores principales. Hay necesidad de revisar estos puntos y determinar la extensión de las responsabilidades tanto para los responsables como para los coparticipes.

Dentro de este título III en la ley (Ley 1278, 2016), refiriéndose a la REP se presenta el Régimen Especial de Gestión de Residuos de Bienes Priorizados, que comprende: 
«i) los bienes de consumo masivo que directa o indirectamente inciden significativamente en la generación de residuos sólidos en volúmenes considerables o ii) que por sus características de peligrosidad requieran de un manejo especial».

Los ACR son residuos de bienes de consumo masivo de volúmenes considerables. Las estimaciones para Lima indican que de los casi 10 millones de habitantes se puede recolectar hasta 60 millones de litros de aceite al año (Mujica, Domínguez y Corrales, 2016). Esto es bastante, más aún si se piensa qué está pasando con esta cantidad de residuos.

En el párrafo 2.4.5. de este documento se presentan los sustentos que señalan la potencial peligrosidad de los ACR tanto para el Ecosistema como para la salud de la comunidad, por lo que estos dos requisitos (volumen y peligrosidad) para que los ACR pasen al régimen especial de gestión de residuos de bienes priorizados están justificados.

El reglamento de la Ley en el Título VII (DS 014, 2017) indica cinco criterios a cumplir para que los productos entren al Régimen especial de bienes priorizados, estos criterios son:

a) La identificación del origen y cadena de valor del residuo sólido del bien.- Los bienes son los aceites comestibles que se fabrican y comercializan para usarlos en la preparación de los alimentos. Sus residuos se generan una vez que se han frito los alimentos y el aceite comienza a cambiar a un color más oscuro, momento en el que debe dejar de usarse en la preparación alimenticia y ser desechado. Es a partir de este momento que se convierte en el residuo ACR. Hasta ese momento el valor agregado que tienen los aceites como alimento ha llegado a su fin; pero estos aceites aún mantienen otro valor que es su poder calorífico, que puede ser usado para producir un combustible renovable y no contaminante, que es el biodiésel. En el proceso productivo se genera también glicerina, que tiene diferentes usos industriales.

Figura 3. Cadena de valor de la producción de biodíésel a partir de los ACR

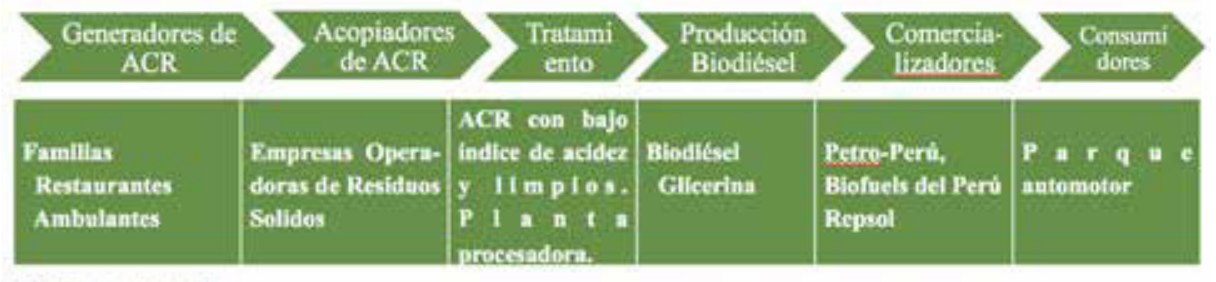

Valor agregado alimenticio

Valor agregado: poder calorífico entre otros

b) Volumen de generación de residuos sólidos asociado al bien. La cantidad de ACR que se genera en Lima, como consecuencia de contar con una población de cerca de 10 millones de habitantes, es de 60,5 millones de litros de aceite (Mujica et al., 2016), como se mencionó al inicio de esta subsección. 
c) Peligrosidad de los residuos sólidos del bien.- Esta calificación ha sido analizada en el punto 2.4.5, haciéndose notar que los ACR pueden llegar a ser potencialmente peligrosos; por los múltiples efectos negativos al suelo, aire y a las fuentes de agua.

d) Posibilidad de valorización de los residuos sólidos del bien.- En la figura 3 se representa la cadena de valor, por la que los ACR son la materia prima para la producción de biodiésel, un biocombustible renovable y que por reglamento (DS 021-2007) debe ser mezclado en un 5\% con el diésel de petróleo, esto es para evitar la contaminación ambiental. Esta mezcla favorece la combustión interna de los motores y estos no liberan partículas pesadas al ambiente, que eran la principal fuente de contaminación del aire por el parque automotor y causa de enfermedades respiratorias. Como consecuencia de esta reglamentación, la calidad del aire en las ciudades del Perú con alto tránsito vehicular ha mejorado considerablemente. Para cumplir con el porcentaje de mezcla del diésel con el biodiésel, el Perú debe importar biodiésel, porque el abastecimiento local no es suficiente; así también se indica que el costo de la materia prima para la producción de biodiésel en el caso de usarse aceites vírgenes es del 75\% del costo total (Lim y Teong, 2010). Por lo tanto, si esta producción se hace con ACR, los costos de producción de biodiésel con ACR disminuye en un porcentaje cercano a los 75\%. En los últimos años los ACR ya se comercializan a 0.60 centavos de Sol por litro. Los estudios sobre las ganancias de esta producción dan cuenta de ser rentables, uno de los estudios presenta un TIR económico de 23,99\% y un VAN económico de aproximadamente 2,6 MM\$, con lo cual, se presume con base a las estimaciones realizadas que es económicamente viable (Godinez, 2013), es más ya hay entidades que están produciendo a pequeña escala en el país.

Por otro lado, están las empresas que están recolectado los aceites, dándoles un tratamiento para mejorar su calidad y exportarlo a otros países donde entran como materia prima para la producción de biodiésel.

e) Alternativas de tecnologías disponibles para su valorización material o energética.- La tecnología más usada a nivel mundial es la misma que para los aceites vírgenes. En el caso de que los ACR sean de baja calidad, estos deben pasar por un acondicionamiento para bajarles el índice de acidez y luego pasar a la etapa de transformación, la que se hace por medio de la reacción química de transesterificación, en la cual el aceite o grasa reacciona con un alcohol en presencia de un catalizador para formar los ésteres del alcohol que se use, que son el biodiésel. Como un coproducto del proceso productivo, se obtiene glicerina que también tiene múltiples usos. Esta técnica ya ha sido implementada en el Perú, desde el año 2000, fruto del trabajo conjunto de la Universidad Nacional Agraria La Molina (UNALM) e Intermediate Technology Development Group - Perú (ITDG) 
(UNALM, 2006), (Coello, Acosta y Velásquez, 2007). Así también empresas como Palmas del Espino, han implementado esta tecnología; pero para los aceites vírgenes de la palma aceitera, algunas municipalidades como la de Surco tienen una planta pequeńa para procesar los ACR de su comunidad. En varias universidades como en la Pontificia Universidad Católica del Perú (PUCP) y otros centros de investigación locales son varios los trabajos sobre el proceso productivo de biodiésel con aceites (Barriga, 2011). Los aspectos químicos (Ballón, 2011), han contado hasta la fecha con un proceso clásico de producción de biodiésel con un rendimiento del 89,6\% de biodiésel en relación al aceite utilizado. También, sobre la gestión de los ACR desde el ámbito académico (Mujica, 2016; Lim y Teong, 2010), municipal (ordenanza de la Municipalidad de Comas para el manejo de los ACR, Municipalidad Distrital de Comas, 2016) y del sector Vivienda y Construcción y Saneamiento (RRR, 2018) se vienen desarrollando acciones a fin de identificar los vacíos a superar y hacer viables modelos de negocio que valoricen los residuos entre ellos los ACR.

Entonces la valorización de los ACR, como materia prima para la producción de biodiésel, se justifica porque atiende a la necesidad de demanda energética del país, siendo el sector de Energía y Minas la entidad competente en biocombustibles. La normativa existente bajo este sector está relacionada a cultivos oleaginosos; pero no hace referencia directa a los ACR, por lo que se torna urgente ante los nuevos usos de los residuos y la importancia que están tomando los ACR dar las pautas regulatorias pertinentes desde este sector.

\section{Conclusión}

Lo presentado muestra que los ACR cuentan con todos los sustentos para ser declarados bienes priorizados, de acuerdo a lo que se dice en el Título III de la ley: «El Ministerio del Ambiente, mediante Decreto Supremo refrendado por los sectores, vinculados, aprueba los bienes priorizados que se encuentran sujetos a este régimen especial de gestión de residuos sólidos, así como los objetivos, las metas y los plazos para la implementación de los sistemas de manejo» (Ley 1278, 2016).

Para los ACR son los sectores de Salud — por las afecciones a la salud que se derivan del mal manejo- y de Energía y Minas — por la importancia que tiene este residuo para la producción de energía-, que junto con el Ministerio del Ambiente deberían declarar prontamente que los ACR sean considerados bienes priorizados sujetos al régimen especial de gestión de residuos sólidos, y de ese modo encaminar acciones para la construcción del sistema de manejo de los ACR de la comunidad, con el soporte legal correspondiente. 


\section{REFERENCIAS}

Ballón, A. (2011). Estudio de los aspectos químicos para el pretratamiento de los aceites comestibles residuales a ser usados para la producción de biodiésel (Tesis de pregrado sin publicar). Pontificia Universidad Católica del Perú, Facultad de Ciencias e Ingeniería, Lima, Perú.

Barriga, E. (2011). Diseño de módulo de transesterificación de una planta piloto para producción de biodiésel a partir de aceites usados de cocina (Tesis de pregrado). Pontificia Universidad Católica del Perú, Facultad de Ciencias e Ingeniería, Lima, Perú.

Coello, J., Acosta, F. y Velásquez, J. (2007). Opciones para la producción de biodiésel a pequeña escala para autoabastecimiento energético en comunidades aisladas de la Amazonía. Lima: ITDG [versión electrónica]. Recuperado el 12/08/2018 de www.hedon. info/docs/Boiling\%20Point_56_Biocombusrtibles_Esp.doc

Dulanto, A. (2017). Análisis de la regulación peruana en materia de responsabilidad extendida del productor en el Reglamento de Residuos de Aparatos Eléctricos y Electrónicos (Tesis de maestría). Pontificia Universidad Católica del Perú, Escuela de Posgrado, Lima, Perú.

Godinez, J.C. (2013). Estudio de pre-factibilidad para la implementación de una planta de biodiesel a base de aceites usados en Lima (Tesis de pregrado). Pontificia Universidad Católica del Perú, Facultad de Ciencias e Ingeniería, Lima, Perú.

Gonzáles. I. y Gonzáles, J.A. (2015). Aceites usados de cocina. Problemática ambiental, incidencias en redes de saneamiento y coste del tratamiento. Bones pràctiques per a la millora de la gestió de residus municipals de Catalunya. Recuperado el 12/08/2018 de http:// residusrecursos.cat/uploads/activitats/docs/20170427092548.pdf

Guillén, M. y Uriarte, P. (2012). Aldehydes contained in edible oils of a very different nature after prolonged heating at frying temperature: Presence of toxic oxygenated a,b unsaturated Aldehydes. Food Chemistry, 131, 915-926. https://doi.org/10.1016/j. foodchem.2011.09.079

Lim, S. y Teong, L.K. (2010). Recent trends, opportunities and challenges of Biodiesel in Malaysia: an overview. Renewable and Sustainable Energy Reviews, 14(3), 938-954. https://doi.org/10.1016/j.rser.2009.10.027

Ministerio del Ambiente (22 de diciembre, 2016). Aprobación de la nueva Ley de Gestión Integral de Residuos Sólidos. [1278]. Recuperado el 12/08/2018 de_http://www. minam.gob.pe/disposiciones/decreto-legislativo-n-1278/

Ministerio del Ambiente (20 de diciembre, 2017). Aprobación del Reglamento del Decreto Legislativo 1278. Recuperado el 12/08/2018 de http://sinia.minam.gob. pe/download/file/fid/60275

Ministerio de Energía y Minas (18 de abril, 2007). Reglamento para la comercialización de Biocombustibles Decreto Supremo 021-2007-EM. Recuperado el 12/08/2018 de http://www.osinergmin.gob.pe/seccion/centro_documental/PlantillaMarco 
LegalBusqueda/Reglamento\%20para\%20la\%20Comercializaci\%C3\%B3n\%20 de\%20Biocombustibles.pdf

Municipalidad Distrital de Comas (08 de junio, 2016). Aprobación del manejo ambiental sostenible de aceites y lubricantes industriales usados y aceites vegetales usados en el distrito de Comas [475]. Recuperado el 12/08/2018 de http://www.municomas.gob. pe/Documentacion/pdf/ORDENANZA\%20N\%20475-2016-MDC.pdf

Mujica, S. E., Domínguez, J. y Corrales, C. (2016). Disposición final de los aceites usados y la calidad. En Congreso de Residuos Sólidos en el Perú. Recuperado el 08/ 09/2018 de http://rrssfc.wixsite.com/v-congreso-residuos/caracterizacion

Resolución Ministerial 363-2005/MINSA. Norma sanitaria para el funcionamiento de restaurantes y servicios afines. Diario Oficial El Peruano, Lima. Perú, 19 de mayo de 2005.

Resolución Legislativa 26234. Convenio de Basilea sobre el control de los movimientos transfronterizos de los desechos peligrosos y su eliminación adoptado por la Conferencia de Plenipotenciarios del 22 de marzo 1989. Recuperado el 03/08/2018 de https:// www.saludarequipa.gob.pe/desa/archivos/Normas_Legales/generales/Basilea.pdf

Proyecto Recuperación y Reutilización Segura de los Recursos (RRR) (2018). Recuperación y Reutilización Segura de Recursos (RRR) en Lima. Recuperado el 03/08/2018 de http://www.rrr.pe/index.php/proyecto-rrr/documentos/category/2-documentos

Tojo, N, (2004). Extended Producer Responsibility as a Driver for Design Change - Utopia or Reality? (Doctoral Dissertation). Lund University, The International Institute for Industrial Environmental Economics, Suecia.

Universidad Nacional Agraria La Molina. Oficina de Relaciones Públicas (11 de marzo, 2006). Opciones para la producción de biodiésel a pequeña escala. Boletín Electrónico de Noticias de la Universidad Nacional Agraria La Molina, 2(73). Lima, Perú. 